Review

\title{
Supersymmetric Quantum Mechanics and Solvable Models
}

\section{Jonathan Bougie $^{1, *}$, Asim Gangopadhyaya ${ }^{1}$, Jeffry Mallow ${ }^{1}$ and Constantin Rasinariu ${ }^{2}$}

${ }^{1}$ Department of Physics, Loyola University Chicago, 1032 W. Sheridan Rd., Chicago, IL 60660, USA; E-Mails: agangop@gmail.com (A.G.); jvmallow@gmail.com (J.M.)

${ }^{2}$ Department of Science and Mathematics, Columbia College Chicago, 600 S. Michigan Ave., Chicago, IL 60605, USA; E-Mail: crasinariu@ colum.edu

* Author to whom correspondence should be addressed; E-Mail: jbougie@ luc.edu; Tel.: +1-773-508-3543; Fax: +1-773-508-3534.

Received: 29 June 2012 ; in revised form: 20 July 2012 / Accepted: 31 July 2012 /

Published: 16 August 2012

\begin{abstract}
We review solvable models within the framework of supersymmetric quantum mechanics (SUSYQM). In SUSYQM, the shape invariance condition insures solvability of quantum mechanical problems. We review shape invariance and its connection to a consequent potential algebra. The additive shape invariance condition is specified by a difference-differential equation; we show that this equation is equivalent to an infinite set of partial differential equations. Solving these equations, we show that the known list of $\hbar$-independent superpotentials is complete. We then describe how these equations could be extended to include superpotentials that do depend on $\hbar$.
\end{abstract}

Keywords: supersymmetry; quantum mechanics; shape invariance

Classification: PACS 03.65.-w, 47.10.-g, 11.30.Pb

\section{Introduction}

Supersymmetric quantum mechanics (SUSYQM) is a generalization of the factorization method commonly used for the harmonic oscillator. The factorization technique begun by Darboux [1] about one hundred years ago, and used by Schrödinger [2-4] in the 1940's and Infeld and Hull in the 1950's [5], could be considered a precursor of SUSYQM. 
The current form of SUSYQM appeared in 1981 [6] as a model of dynamical symmetry breaking. It was developed further by the authors of [7,8] among others. This simplified model turned out to have important applications in quantum mechanics.

In the next section, we will describe the general formalism of SUSYQM and in Section 3 we introduce the shape invariance condition that makes a potential solvable. Section 4 is dedicated to a description of potential algebra and its connection to shape invariance. In Section 5 we will describe a method for determining solutions of the translational shape invariance condition. We will then conclude with the analysis of recently discovered shape invariant potentials that are inherently functions of $\hbar$.

\section{Supersymmetric Quantum Mechanics}

Throughout this paper, we use units such that $2 m=1$. For the harmonic oscillator, the Hamiltonian [9] $H_{h o}=-\hbar^{2} \frac{d^{2}}{d x^{2}}+\frac{1}{4} \omega^{2} x^{2}-\frac{1}{2} \hbar \omega$ is factorized into $\mathcal{A}^{+} \equiv-\hbar \frac{d}{d x}+\frac{1}{2} \omega x$ and $\mathcal{A}^{-} \equiv \hbar \frac{d}{d x}+\frac{1}{2} \omega x$. Similarly, in the SUSYQM formalism [10,11] a general Hamiltonian $H_{-}=-\hbar^{2} \frac{d^{2}}{d x^{2}}+V_{-}(x, a)$ is written as a product of $\mathcal{A}^{+} \equiv-\hbar \frac{d}{d x}+W(x, a)$ and $\mathcal{A}^{-} \equiv \hbar \frac{d}{d x}+W(x, a)$, where the function $W(x, a)$ is known as the superpotential. The product $\mathcal{A}^{+} \mathcal{A}^{-}$ is then given by

$$
\mathcal{A}^{+} \mathcal{A}^{-}=\left(-\hbar \frac{d}{d x}+W(x, a)\right)\left(\hbar \frac{d}{d x}+W(x, a)\right)=-\hbar^{2} \frac{d^{2}}{d x^{2}}+W^{2}(x, a)-\hbar \frac{d W(x, a)}{d x}
$$

Thus, the product $\mathcal{A}^{+} \mathcal{A}^{-}$indeed reproduces the Hamiltonian $H_{-}$above, provided the potential $V_{-}(x, a)$ is related to the superpotential $W(x, a)$ by $V_{-}(x, a)=W^{2}(x, a)-\hbar \frac{d W(x, a)}{d x}$. The product $\mathcal{A}^{-} \mathcal{A}^{+}$ produces another Hamiltonian $H_{+}=-\hbar^{2} \frac{d^{2}}{d x^{2}}+V_{+}(x, a)$ with $V_{+}(x, a)=W^{2}(x, a)+\hbar \frac{d W(x, a)}{d x}$. To see the underlying supersymmetry of this formalism, let us construct a generator $Q^{-}$and its adjont $Q^{+}$by:

$$
Q^{-}=\left(\begin{array}{cc}
0 & 0 \\
\mathcal{A}^{-} & 0
\end{array}\right) ; \text { and } Q^{+}=\left(\begin{array}{cc}
0 & \mathcal{A}^{+} \\
0 & 0
\end{array}\right)
$$

Operators $Q^{-}$and $Q^{+}$generate the following supersymmetry algebra:

$$
\left\{Q^{-}, Q^{-}\right\}=\left\{Q^{+}, Q^{+}\right\}=0 ; \text { and }\left\{Q^{-}, Q^{+}\right\}=H \equiv\left(\begin{array}{cc}
\mathcal{A}^{+} \mathcal{A}^{-} & 0 \\
0 & \mathcal{A}^{-} \mathcal{A}^{+}
\end{array}\right)
$$

The groundstate energy of this Hamiltonian is then given by

$$
\left.\left.\left\langle\Psi_{0}|H| \Psi_{0}\right\rangle=\left\langle\Psi_{0}\left|\left\{Q^{-}, Q^{+}\right\}\right| \Psi_{0}\right\rangle=\left|Q^{+}\right| \Psi_{0}\right\rangle\left.\right|^{2}+\left|Q^{-}\right| \Psi_{0}\right\rangle\left.\right|^{2} \geq 0
$$

Thus, the non-vanishing of the groundstate energy implies that either $Q^{+}\left|\Psi_{0}\right\rangle \neq 0$ or $Q^{-}\left|\Psi_{0}\right\rangle \neq 0$, and hence signals the spontaneous breaking of the supersymmetry. We therefore require that $\left\langle\Psi_{0}|H| \Psi_{0}\right\rangle=0$ to preserve unbroken supersymmetry.

The Hamiltonians $H_{+}$and $H_{-}$are intertwined; i.e., $\mathcal{A}^{+} H_{+}=H_{-} \mathcal{A}^{+}$and $\mathcal{A}^{-} H_{-}=H_{+} \mathcal{A}^{-}$. This leads to the following relationships among their eigenvalues and eigenfunctions [12]

$$
E_{n+1}^{-}=E_{n}^{+} ; \quad \frac{\mathcal{A}^{-}}{\sqrt{E_{n}^{+}}} \psi_{n+1}^{(-)}=\psi_{n}^{(+)} \quad \text { and } \frac{\mathcal{A}^{+}}{\sqrt{E_{n}^{+}}} \psi_{n}^{(+)}=\psi_{n+1}^{(-)} ; \quad n=0,1,2 \cdots
$$


Since $H_{ \pm}$are products of the operator $\mathcal{A}^{-}$and its adjoint $\mathcal{A}^{+}$, their eigenvalues are either zero or positive [13]. The ground state eigenvalue of one of these Hamiltonians must be zero in order to have unbroken supersymmetry. Without loss of generality, we choose that Hamiltonian to be $H_{-}$. Thus, we have

$\mathcal{A}^{+} \mathcal{A}^{-} \psi_{0}^{(-)}=0$; which is satisfied when $\mathcal{A}^{-} \psi_{0}^{(-)}=0$, i.e. $\psi_{0}^{(-)}(x, a)=N e^{-\frac{1}{\hbar} \int_{x_{0}}^{x} W(y, a) d y}$

where $x_{0}$ is an arbitrary point in the domain and $N$ is the normalization constant that depends on the choice of $x_{0}$. Thus, if $\psi_{0}^{(-)}$is a normalizable groundstate, we have a system with unbroken supersymmetry. Its groundstate $E_{0}^{-}$is zero and the operator $\mathcal{A}^{-}$annihilates the corresponding eigenstate $\psi_{0}^{(-)}$. For all higher states of $H_{-}$, as indicated in Equation (5), there is an one-to-one correspondence with the states of $H_{+}$.

\subsection{Example}

Consider a system described by the superpotential $W(x, b)=-b \cot x$, defined over the domain $(0, \pi)$. Corresponding partner potentials are given by $V_{ \pm}(x, b)=b(b \pm \hbar) \operatorname{cosec}^{2} x-b^{2}$. This is a rather complicated potential, and rarely analyzed in quantum mechanics courses. However, for $b=\hbar$, the potential $V_{-}(x, \hbar)$ reduces to the infinite square well, with the bottom of the potential at $-\hbar^{2}$ and zero groundstate energy. We know that the corresponding eigenstates are given by $\psi_{n}^{(-)}=\sin (n x)$ and eigenvalues $E_{n}^{-}=n^{2} \hbar^{2}$. Thus, using the familiarity with the relatively simpler potential $V_{-}$, we are able to derive all of the eigenvalues and eigenfunctions of $V_{+}$. Then the inter-relations expressed through Equation (5) enable us to determine eigenvalues and eigenfunctions of $V_{+}(x, \hbar)=2 \hbar^{2} \operatorname{cosec}^{2} x-\hbar^{2}$.

Although we assume that our Hamiltonians are hermitian, hermiticity is not necessary to generate real eigenvalues. Replacing the sufficient but not necessary condition of hermiticity with the weaker condition of PT symmetry, has led to the discovery of new potentials with real energy eigenvalues [14-16]. Considerable work has been done on the study of SI potentials with PT symmetry. In reference [17], the author examined the shape invariant hyperbolic Rosen-Morse potential as a case of exact solvability with PT invariance. In reference [18], the Scarf II potential was shown as an example of spontaneous PT-symmetry-breaking. The case of a square well with discrete PT symmetry but with intervals of non-hermiticity was shown in reference [19] to produce real eigenvalues. Authors of [20] constructed the spectrum of a square well of imaginary strength, to obtain the hierarchy of SI potentials, while authors of [21] constructed a set of solvable rational potentials, and related the PT symmetry condition to the condition that they be free of singularities. However, in this paper we limit ourselves to the case of hermitian Hamiltonians, i.e., real $W(x, a)$.

The remainder of this manuscript is devoted to the study of the shape invariance condition and its solutions.

\section{Shape Invariance in Supersymmetric Quantum Mechanics}

If the superpotential $W\left(x, a_{i}\right)$ of a system obeys the condition

$$
W^{2}\left(x, a_{i}\right)+\hbar \frac{d W\left(x, a_{i}\right)}{d x}+g\left(a_{i}\right)=W^{2}\left(x, a_{i+1}\right)-\hbar \frac{d W\left(x, a_{i+1}\right)}{d x}+g\left(a_{i+1}\right)
$$


where $a_{i+1}=\eta\left(a_{i}\right)$, the system is called shape invariant [5,22-24]. Various forms of the function $\eta$ define classes of shape invariance. The most commonly discussed classes are:

1. Translational or Additive Shape Invariance:

$$
a_{i+1}=\eta\left(a_{i}\right)=a_{i}+\hbar
$$

2. Multiplicative or Scaling Shape Invariance [25,26]: $a_{i+1}=\eta\left(a_{i}\right)=r a_{i}$ where $0<r<1$;

3. Cyclic Shape Invariance [27]: $\quad a_{i+n}=\eta^{n}\left(a_{i}\right)=a_{i}$.

From Equation (7) it follows that for a shape invariant system, the partner potentials $V_{+}\left(x, a_{i}\right)$ and $V_{-}\left(x, a_{i+1}\right)$ differ only by values of parameter $a_{i}$ and additive constants $g\left(a_{i}\right)$. In particular,

$$
V_{+}\left(x, a_{0}\right)+g\left(a_{0}\right)=V_{-}\left(x, a_{1}\right)+g\left(a_{1}\right)
$$

In terms of operators $\mathcal{A}^{ \pm}$, the shape invariance condition becomes

$$
\mathcal{A}^{-}\left(x, a_{0}\right) \mathcal{A}^{+}\left(x, a_{0}\right)-\mathcal{A}^{+}\left(x, a_{1}\right) \mathcal{A}^{-}\left(x, a_{1}\right)=g\left(a_{1}\right)-g\left(a_{0}\right)
$$

As we will see in Section 4, Equation (9) implies that for every shape invariant system, there is always an underlying potential algebra [28-32] that guarantees its solvability. In the rest of this section, we will show how shape invariance enables us to find the eigenvalues and eigenfunctions of the system.

\subsection{Determination of Eigenvalues}

From Equation (9), we see that Hamiltonians $H_{+}\left(x, a_{0}\right)$ and $H_{-}\left(x, a_{1}\right)$ differ only by the constant $g\left(a_{1}\right)-g\left(a_{0}\right)$. We already know that $E_{0}^{-}\left(a_{0}\right)=0$. Let us determine the first excited state of $H_{-}$; i.e., $E_{1}^{-}\left(a_{0}\right)$. Hence, using $E_{0}^{-}\left(a_{1}\right)=0$ [33], we find that the energy $E_{1}^{-}\left(a_{0}\right)$ of the first excited state of $H_{-}\left(x, a_{0}\right)$ and $E_{0}^{+}\left(a_{0}\right)$ of the groundstate of $H_{+}\left(x, a_{0}\right)$ both are given by $g\left(a_{1}\right)-g\left(a_{0}\right)$. Similarly, to determine $E_{2}^{-}\left(a_{0}\right)$, we use the isospectrality condition (5) to relate it to $E_{1}^{+}\left(a_{0}\right)$. But by the shape invariance condition (9), $E_{1}^{+}\left(a_{0}\right)=E_{1}^{-}\left(a_{1}\right)+g\left(a_{1}\right)-g\left(a_{0}\right)$. Following the method used for determining $E_{1}^{-}\left(a_{0}\right)$, we find $E_{1}^{-}\left(a_{1}\right)=g\left(a_{2}\right)-g\left(a_{1}\right)$, and hence, $E_{2}^{-}\left(a_{0}\right)=g\left(a_{2}\right)-g\left(a_{1}\right)+g\left(a_{1}\right)-g\left(a_{0}\right)=g\left(a_{2}\right)-g\left(a_{0}\right)$. Extending this argument to higher excited states of $H_{-}\left(x, a_{0}\right)$, we get

$$
E_{n}^{(-)}\left(a_{0}\right)=g\left(a_{n}\right)-g\left(a_{0}\right) \text { for } n \geq 0
$$

\subsection{Determination of Eigenfunctions}

Again from Equation (9), we see that since $H_{+}\left(x, a_{0}\right)$ and $H_{-}\left(x, a_{1}\right)$ only differ by the constant $g\left(a_{1}\right)-g\left(a_{0}\right)$, they must have common eigenfunctions. Hence, from Equation (6), we have $\psi_{0}^{(+)}\left(x, a_{0}\right)=\psi_{0}^{(-)}\left(x, a_{1}\right) \sim e^{-\frac{1}{\hbar} \int^{x} W\left(y, a_{1}\right) d y}$. Then the isospectrality condition (5), requires $\psi_{1}^{(-)}\left(x, a_{0}\right) \sim \mathcal{A}^{+}\left(a_{0}\right) e^{-\frac{1}{\hbar} \int^{x} W\left(y, a_{1}\right) d y}$. Iterating this procedure, we obtain

$$
\begin{aligned}
\psi_{n}^{(-)}\left(x, a_{0}\right) & \sim \mathcal{A}^{+}\left(a_{0}\right) \mathcal{A}^{+}\left(a_{1}\right) \cdots \mathcal{A}^{+}\left(a_{n-1}\right) \psi_{0}^{(-)}\left(x, a_{n}\right) \\
& \sim \mathcal{A}^{+}\left(a_{0}\right) \mathcal{A}^{+}\left(a_{1}\right) \cdots \mathcal{A}^{+}\left(a_{n-1}\right) e^{-\frac{1}{\hbar} \int^{x} W\left(y, a_{n}\right) d y}
\end{aligned}
$$


Thus, for a system with a given shape invariant superpotential, the eigenvalues and eigenfunctions can be determined analytically. This result makes it very important to find all such potentials. In the past, researchers had found a list of additive shape invariant potentials [10,11], mostly by trial and error [34,35]. In Section 5, we will discuss how to find solutions of Equation (9) for the additive case. Before that, however, in the next section we will show why the shape invariance condition leads to solvability.

\section{Shape Invariance and Potential Algebra}

We will now show that the symmetry behind the shape invariance is essential in building the algebraic structures known as potential algebras. As we will show below, a potential algebra is in general a deformation of a three-dimensional Lie algebra, whose representations yield the spectrum of the corresponding shape invariant system.

\subsection{Building the Algebra}

The starting point of our construct is the shape invariance condition given by Equation (9), which we rewrite as

$$
\mathcal{A}^{+}\left(x, a_{1}\right) \mathcal{A}^{-}\left(x, a_{1}\right)-\mathcal{A}^{-}\left(x, a_{0}\right) \mathcal{A}^{+}\left(x, a_{0}\right)=f\left(a_{0}\right)
$$

where $f\left(a_{0}\right)=g\left(a_{0}\right)-g\left(a_{1}\right) \equiv g\left(a_{0}\right)-g\left(\eta\left(a_{0}\right)\right)$, and $\eta$ is a function that models the change of parameter $a_{1}=\eta\left(a_{0}\right)$. For example, if the change of parameter is a translation, $a_{1} \equiv \eta\left(a_{0}\right)=a_{0}+s$.

The left hand side of Equation (12) resembles a commutation relation. This suggests that we use $\mathcal{A}^{-}$and $\mathcal{A}^{+}$to build the generators of the potential algebra. To transform the above shape invariance condition into an exact commutator, we replace operators $\mathcal{A}^{-}\left(x, a_{0}\right)$ and $\mathcal{A}^{+}\left(x, a_{0}\right)$ by

$$
\begin{aligned}
& \mathcal{A}^{-}\left(x, a_{0}\right) \quad \mapsto J_{-} \equiv \hat{\mathcal{A}}^{-}\left(x, \chi\left(i \partial_{\phi}\right)\right) \mathrm{e}^{-i s \phi}, \text { and } \\
& \mathcal{A}^{+}\left(x, a_{0}\right) \quad \mapsto J_{+} \equiv\left(J_{-}\right)^{\dagger}=\mathrm{e}^{i s \phi} \hat{\mathcal{A}}^{+}\left(x, \chi\left(i \partial_{\phi}\right)\right)
\end{aligned}
$$

where $s$ is a constant parameter, $\phi$ is an auxiliary variable, and $\partial_{\phi} \equiv \frac{\partial}{\partial \phi}$. The function $\chi$ will be appropriately chosen to emulate the relationship between parameters $a_{0}$ and $a_{1}$. Thus, to generate $J_{-}$, we multiplied the operator $\mathcal{A}^{-}\left(x, a_{0}\right)$ from right by $\mathrm{e}^{-i s \phi}$ and replaced the parameter $a_{0}$ by the differential operator $\chi\left(i \partial_{\phi}\right)$. If we now compute the commutator between operators $J_{+}$and $J_{-}$, we find [36]

$$
\begin{aligned}
{\left[J_{+}, J_{-}\right] } & =J_{+} J_{-}-J_{-} J_{+} \\
& =\mathrm{e}^{i s \phi} \hat{\mathcal{A}}^{+}\left(z, \chi\left(i \partial_{\phi}\right)\right) \hat{\mathcal{A}}^{-}\left(x, \chi\left(i \partial_{\phi}\right)\right) \mathrm{e}^{-i s \phi}-\hat{\mathcal{A}}^{-}\left(x, \chi\left(i \partial_{\phi}\right)\right) \hat{\mathcal{A}}^{+}\left(x, \chi\left(i \partial_{\phi}\right)\right) \\
& =\hat{\mathcal{A}}^{+}\left(x, \chi\left(i \partial_{\phi}+s\right)\right) \hat{\mathcal{A}}^{-}\left(x, \chi\left(i \partial_{\phi}+s\right)\right)-\hat{\mathcal{A}}^{-}\left(x, \chi\left(i \partial_{\phi}\right)\right) \hat{\mathcal{A}}^{+}\left(x, \chi\left(i \partial_{\phi}\right)\right)
\end{aligned}
$$

Observe now that the right hand side of Equation (14) matches the left hand side of Equation (12) provided that we make the following mappings

$$
a_{0} \mapsto \chi\left(i \partial_{\phi}\right) \quad a_{1} \mapsto \chi\left(i \partial_{\phi}+s\right)
$$


Since we know that $a_{1}=\eta\left(a_{0}\right)$, these mappings require that the function $\chi\left(i \partial_{\phi}\right)$ satisfy

$$
\chi\left(i \partial_{\phi}+s\right)=\eta\left(\chi\left(i \partial_{\phi}\right)\right)
$$

Let us look at some examples to illustrate this procedure.

- Translation: $a_{1} \equiv \eta\left(a_{0}\right)=a_{0}+s$

If the change of parameters is a translation, then the function $\chi$ that models it is the identity function

$$
\chi(z)=z
$$

We have $\chi(z+s)=z+s=\chi(z)+s$, which gives the desired change of parameters.

- Scaling: $a_{1} \equiv \eta\left(a_{0}\right)=r a_{0}$

For shape invariant potentials characterized by a scaling change of parameters, the corresponding function $\chi$ is the exponential

$$
\chi(z)=\mathrm{e}^{z}
$$

Indeed $\chi(z+s)=\mathrm{e}^{z+s}=\mathrm{e}^{s} \mathrm{e}^{z}=r \chi(z)$ where we denoted $\mathrm{e}^{s}=r$.

- Cyclic: $a_{0}, a_{1}=\eta\left(a_{0}\right), a_{2}=\eta\left(a_{1}\right), \ldots, a_{k-1}=\eta\left(a_{k-2}\right), a_{k}=\eta\left(a_{k-1}\right)=a_{0}$

Cyclic potentials form a series of shape invariant potentials that repeats after a cycle of $k$ iterations. These potentials appear also in connection with the dressing chain formalism [37]. To satisfy the cyclic parameter change, the function $\eta$ should obey $\eta(\eta(\ldots \eta(z) \ldots)) \equiv \eta^{k}(z)=z$. The projective map $\eta(z)=(\alpha z+\beta) /(\gamma z+\delta)$ with specific constraints [38] on the parameters $\alpha, \beta, \gamma$, and $\delta$ satisfies such a condition [27]. The function $\chi$ satisfying Equation (16) in this case is given by

$$
\chi(z)=\frac{\left(\lambda_{1}-\delta\right) \lambda_{1}^{z / s}+\left(\lambda_{2}-\delta\right) \lambda_{2}^{z / s} B(z)}{\gamma\left[\lambda_{1}^{z / s}+\lambda_{2}^{z / s} B(z)\right]}
$$

where $\lambda_{1,2}$ are solutions of the equation $(\lambda-\alpha)(\lambda-\delta)-\beta \delta=0$ and $B(z)$ is an arbitrary periodic function of $z$ with period $k$.

- Other choices of parameters follow from more complicated choices for $\chi(z)$. For example taking

$$
\chi(z)=\exp \left(e^{z}\right)
$$

we obtain the change of parameters: $a_{1}=a_{0}^{2}$. 
Now, let us get back to the building of the potential algebra. In terms of operators $J_{+}$and $J_{-}$, the shape invariance condition (12) becomes:

$$
\left[J_{+}, J_{-}\right]=f\left(\chi\left(i \partial_{\phi}\right)\right)
$$

Thus, the commutation relation of operators $J_{+}(x, \phi)$ and $J_{-}(x, \phi)$ generates an operator $f\left(\chi\left(i \partial_{\phi}\right)\right)$ that has no $x$-dependence. If we now define a third operator $J_{3}$ in terms of the operator $i \partial_{\phi}$, the shape invariance condition becomes simply one of the commutation relations of the potential algebra. In particular, if we define

$$
J_{3}=k-\frac{i}{s} \partial_{\phi}
$$

where $k$ is an arbitrary constant, the three commutators among these generators are given by [39]

$$
\left[J_{3}, J_{+}\right]=J_{+}, \quad\left[J_{3}, J_{-}\right]=-J_{-}, \quad\left[J_{+}, J_{-}\right]=f\left(\chi\left(i \partial_{\phi}\right)\right) \equiv F\left(J_{3}\right)
$$

Putting together the above results, we arrive at the following

Lemma: To any shape invariant system characterized by

$$
\mathcal{A}^{+}\left(x, a_{1}\right) \mathcal{A}^{-}\left(x, a_{1}\right)-\mathcal{A}^{-}\left(x, a_{0}\right) \mathcal{A}^{+}\left(x, a_{0}\right)=f\left(a_{0}\right), a_{1}=\eta\left(a_{0}\right)
$$

for which we can find a function $\chi$ such that $\chi(z+s)=\eta(\chi(z))$ for arbitrary parameters $z$ and $s$, we can associate an algebra [40] generated by

$$
\begin{aligned}
J_{+} & =\mathrm{e}^{i s \phi} \hat{\mathcal{A}}^{+}\left(z, \chi\left(i \partial_{\phi}\right)\right) \\
J_{-} & =\hat{\mathcal{A}}^{-}\left(z, \chi\left(i \partial_{\phi}\right)\right) \mathrm{e}^{-i s \phi} \\
J_{3} & =k-\frac{i}{s} \partial_{\phi}
\end{aligned}
$$

satisfying the commutation relations

$$
\left[J_{3}, J_{+}\right]=J_{+}, \quad\left[J_{3}, J_{-}\right]=-J_{-}, \quad\left[J_{+}, J_{-}\right]=F\left(J_{3}\right)
$$

where

$$
F\left(J_{3}\right)=f\left(\chi\left(s k-s J_{3}\right)\right)
$$

The function $f$ in Equation (29) is given by the shape invariance condition (24), while the function $\chi$ satisfies the compatibility equation: $\chi\left(i \partial_{\phi}+s\right)=\eta\left(\chi\left(i \partial_{\phi}\right)\right)$, where $\eta$ models the change of parameter $a_{1}=\eta\left(a_{0}\right)$ of Equation (24). 
As an example let us build the potential algebra corresponding to the Pöschl-Teller II potential. The potential

$$
V_{-}(x, l)=-l(l+\hbar) \operatorname{sech}^{2} x+l^{2}
$$

is generated by the superpotential $W(x, l)=l \tanh x$, through $V_{-}=W^{2}-\hbar W^{\prime}$, where $W^{\prime} \equiv \frac{\partial W}{\partial x}$. Its supersymmetric partner $V_{+}=W^{2}+\hbar W^{\prime}$ is given by

$$
V_{+}(x, l)=-l(l-\hbar) \operatorname{sech}^{2} x+l^{2}
$$

The shape invariance is now evident if we observe that $V_{+}(x, l)=V_{-}(x, l-\hbar)+(2 l-\hbar)$. Therefore, in terms of $\mathcal{A}^{+}$and $\mathcal{A}^{-}$operators, the shape invariance (24) for the Pöschl-Teller II potential reads

$$
A^{+}(x, l-\hbar) A^{-}(x, l-\hbar)-A^{-}(x, l) A^{+}(x, l)=\hbar-2 l
$$

Now we can identify the main objects of our model and build the corresponding algebra:

1. The parameters of the model are $a_{0}=\alpha-l$ and $a_{1}=\alpha-l+\hbar$, where $\alpha$ is an arbitrary positive constant greater than $l$ so that $a_{0}$ is a positive quantity;

2. The change of parameter $a_{1}=\eta\left(a_{0}\right)$ is thus given by $a_{1}=a_{0}+\hbar$. This is a translational change of parameter $a_{1}=a_{0}+s$ with the translation parameter $s=\hbar$. Translation implies that the function $\chi$ satisfying (16) is the identity function $\chi(z)=z$;

3. From the concrete shape invariance condition (32) of this potential we get $f\left(a_{0}\right)=\hbar-2\left(\alpha-a_{0}\right)=\hbar-2 \alpha+2 a_{0}$. Then, the function $F\left(J_{3}\right)$ is given by $\left.F\left(J_{3}\right)=f\left(\chi\left(s k-s J_{3}\right)\right)\right)=f\left(J_{3}-k\right)=\hbar-2 \alpha+2\left(J_{3}-k\right)=2 J_{3}$ if we choose the arbitrary constant $k=\frac{1}{2} \hbar-\alpha$;

4. Defining $J_{ \pm}$and $J_{3}$ as prescribed by Equations (25) and (27), we obtain the differential realization of the algebra's generators:

$$
J_{+}=\mathrm{e}^{-i \phi}\left(-\frac{d}{d x}+i \partial_{\phi} \tanh x\right), \quad J_{-}=\left(\frac{d}{d x}+i \partial_{\phi} \tanh x\right) \mathrm{e}^{i \phi}, \quad J_{3}=\frac{1}{2} \hbar-\alpha+i \partial_{\phi}
$$

satisfying the commutation relations $\left[J_{3}, J_{ \pm}\right]= \pm J_{ \pm}$, and $\left[J_{+}, J_{-}\right]=2 J_{3}$. Thus, shape invariance of this system implies that the system has a potential algebra given by $s u(2)[41,42]$.

\subsection{Obtaining the Energy Spectrum from Algebra Representations}

Once we know the potential algebra for a given potential, we can use its representations to obtain the energy spectrum for the Hamiltonian. Using Equations (25) and (26), we observe that

$$
J_{+} J_{-}=\hat{\mathcal{A}}^{+}\left(x, \chi\left(i \partial_{\phi}+s\right)\right) \hat{\mathcal{A}}^{-}\left(x, \chi\left(i \partial_{\phi}+s\right)\right)
$$


From the reciprocal of the mapping Equation (13), we obtain $\hat{\mathcal{A}}^{+}\left(x, \chi\left(i \partial_{\phi}+s\right)\right) \hat{\mathcal{A}}^{-}\left(z, \chi\left(i \partial_{\phi}+s\right)\right) \mapsto$ $\mathcal{A}^{+}\left(x, a_{1}\right) \mathcal{A}^{-}\left(x, a_{1}\right)=H_{-}\left(x, a_{1}\right)$. Consequently, the spectrum of the operator $J_{+} J_{-}$gives the spectrum of the Hamiltonian. To find the concrete values for the energy, we need to know the action of individual operators $J_{+}$and $J_{-}$respectively on a set of eigenvectors of the operator $J_{3}$. In this general case, $J_{+}, J_{-}$ and $J_{3}$ commute with the Casimir operator given by

$$
C=J_{-} J_{+}+G\left(J_{3}\right)=J_{+} J_{-}+G\left(J_{3}-1\right)
$$

with the function $G$ (defined up to an additive constant) such that

$$
F\left(J_{3}\right)=G\left(J_{3}\right)-G\left(J_{3}-1\right)
$$

It can be explicitly checked that $C$ does indeed commute with $J_{+}, J_{-}$and $J_{3}$ [41]. In a basis in which $J_{3}$ and $C$ are diagonal, $J_{ \pm}$play the role of raising and lowering operators, respectively. Operating on an arbitrary eigenstate $|q\rangle$ we have

$$
\begin{aligned}
J_{3}|q\rangle & =q|q\rangle \\
J_{-}|q\rangle & =a(q)|q-1\rangle \\
J_{+}|q\rangle & =a^{\star}(q+1)|q+1\rangle
\end{aligned}
$$

where we have used $J_{+}=\left(J_{-}\right)^{\dagger}$.

Keeping in mind that $H_{-}\left(x, a_{1}\right) \mapsto J_{+} J_{-}$, and observing that $J_{+} J_{-}|q\rangle=|a(q)|^{2}|q\rangle$, we see that in order to find the energies of the system one must find the coefficients $a(q)$. If we apply the third commutation relation of Equation (23) to $|q\rangle$, we obtain using Equation (36)

$$
|a(q)|^{2}-|a(q+1)|^{2}=G(q)-G(q-1)
$$

Next, we will determine the allowed values of $q$ and the corresponding values $a(q)$. Let us say $q=q_{\min }$ corresponds to the lowest state in a given representation of the algebra. This implies that $J_{-}\left|q_{\text {min }}\right\rangle=a\left(q_{\text {min }}\right)|q-1\rangle=0$, which means $a\left(q_{\text {min }}\right)=0$. From Equation (38) we get

$$
a\left(q_{\min }+1\right)^{2}=G\left(q_{\min }-1\right)-G\left(q_{\min }\right)
$$

Iterating this procedure we can generate a general formula for $a(q)$

$$
a\left(q_{\text {min }}+n\right)^{2}=G\left(q_{\text {min }}-1\right)-G\left(q_{\text {min }}+n-1\right) \quad n=1,2, \cdots
$$

Substituting $q_{\min }+n=q$ leads to

$$
a(q)^{2}=G(q-n-1)-G(q-1) \quad n=1,2, \cdots
$$


The profile of $G(q)$ determines the dimension of the representation. For example, let us consider the two cases presented in Figure 1.

Figure 1. Generic behaviors of $G(q)$. Case (a) corresponds to a finite, and (b) to an infinite representation of the potential algebra.

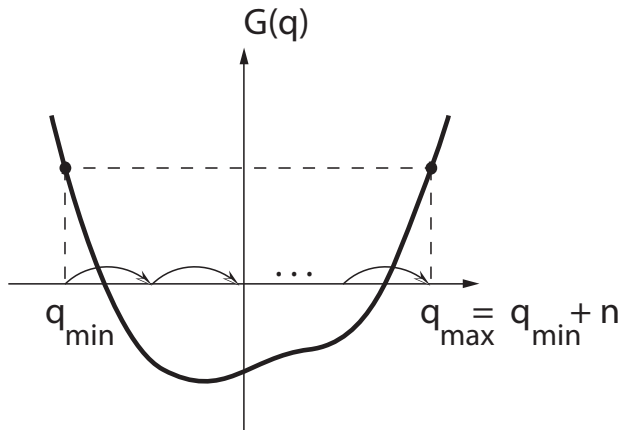

(a)

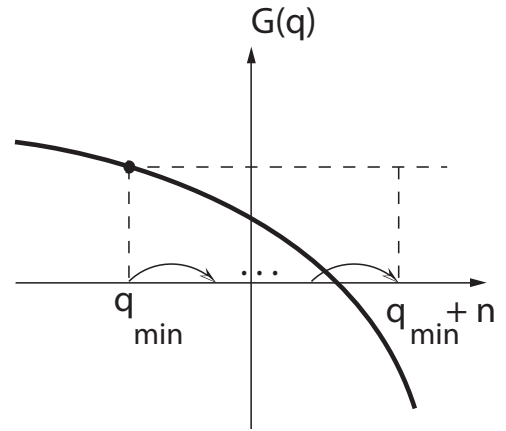

(b)

One obtains the finite dimensional representations of Figure 1a, by starting from a point on the $G(q)$ vs. $q$ graph corresponding to $q=q_{\text {min }}$, and moving in integer steps parallel to the $q$-axis to the point corresponding to $q=q_{\max }$. At the end points, $a\left(q_{\min }\right)=a\left(q_{\max }+1\right)=0$, and we get a finite representation. If $G(q)$ is decreasing monotonically, as in Figure 1b, there exists only one end point at $q=q_{\text {min }}$. Starting from $q_{\text {min }}$ the value of $q$ can be increased in integer steps up to infinity. In this case we have an infinite dimensional representation. As in the finite case, $q_{\min }$ labels the representation. The difference is that here $q_{\min }$ takes continuous values. Similar arguments apply for a monotonically increasing function $G(q)$. Having the representation of the algebra associated with a characteristic model, we obtain, using Equation (41), the complete spectrum of the system.

For example, let us consider the scaling change of parameters $a_{1}=r a_{0}$. Consider the simple choice $f\left(a_{0}\right)=-r_{1} a_{0}$, where $r_{1}$ is a constant. This choice generates self-similar potentials studied in references [25,26,44]. In this case, combining Equation (18) with Equation (28) yields:

$$
\left[J_{3}, J_{ \pm}\right]= \pm J_{ \pm} \quad ; \quad\left[J_{+}, J_{-}\right]=-r_{1} \exp \left(-s J_{3}\right)
$$

which is a deformation of the standard $s o(2,1)$ Lie algebra.

For this case, from Equations (42) and (36) one gets

$$
G(q)=\frac{r_{1}}{e^{s}-1} e^{-s q}=-\frac{r_{1}}{1-r} r^{-q} \quad ; \quad r=e^{s}
$$

Note that for scaling problems [25], one requires $0<r<1$, which leads to $s<0$. From the monotonically decreasing profile of the function $G(q)$, it follows that the unitary representations of this algebra are infinite dimensional. If we label the lowest weight state of the operator $J_{3}$ by $q_{\text {min }}$, then 
$a\left(q_{\min }\right)=0$. Without loss of generality we can choose the coefficients $a(q)$ to be real. Then one obtains from (38) for an arbitrary $q=q_{\text {min }}+n, n=0,1,2, \ldots$

$$
a^{2}(q)=G(q-n-1)-G(q-1)=r_{1} \frac{r^{n}-1}{r-1} r^{1-h}
$$

The spectrum of the Hamiltonian $H_{-}\left(x, a_{1}\right)$ is given by

$$
H_{-}|q\rangle=a^{2}(q)|q\rangle=r_{1} \frac{r^{n}-1}{r-1} r^{1-q}|q\rangle
$$

Therefore, the eigenenergies are

$$
E_{n}(q)=r_{1} \alpha(q) \frac{r^{n}-1}{r-1} \quad ; \quad \alpha(q) \equiv r^{1-q}
$$

in agreement with the known results [25].

\section{How Do We Find Additive Shape Invariant Superpotentials?}

Since we have demonstrated the value of shape-invariant superpotentials, the question now becomes how to find such superpotentials. This question is equivalent to asking how to solve the difference-differential Equation (9) to find the list of desired superpotentials $W$. For this section, we will restrict ourselves to considering cases of translational shape invariance. Before we embark on solving this equation, let us first note that quantum mechanical potentials generally have terms of two very different orders: One "large" and another "small". For example, the classical and quantum potentials for the radial oscillator system are $\frac{1}{4} \omega r^{2}+\frac{L^{2}}{r^{2}}$ and $\frac{1}{4} \omega r^{2}+\frac{\ell(\ell+1) \hbar^{2}}{r^{2}}$ respectively. To make the transition from the quantum to the classical system, one takes the limit $\lim _{\{\ell \rightarrow \infty, \hbar \rightarrow 0\}}$ with the constraint that $\ell \cdot \hbar \rightarrow L$. Thus, the quantum Hamiltonian can be written as $\frac{1}{4} \omega r^{2}+\frac{L^{2}}{r^{2}}+\frac{\hbar L}{r^{2}}$. This shows that in quantum mechanics, the potential generally has one small term that depends on $\hbar$ [43]. In SUSYQM, since the potential is given by $W^{2}(x, a)-\hbar \frac{d W(x, a)}{d x}$, the derivative term always brings in a factor of $\hbar$, even if the superpotential is independent of $\hbar$. In the following analysis, as we determine how to solve Equation (9) to find shape invariant superpotentials, we will consider the cases of $\hbar$-independent and $\hbar$-dependent superpotentials separately.

\subsection{Known $\hbar-I n d e p e n d e n t ~ S h a p e ~ I n v a r i a n t ~ S u p e r p o t e n t i a l s$}

We begin our discussion of known shape invariant systems by considering only superpotentials that do not depend explicitly on $\hbar$, which we call "conventional" superpotentials. In Table 1 we list the known "conventional" superpotentials that meet this criterion.

Previous work [45-47] has proven that this list of conventional shape-invariant superpotentials is complete. We now show a new proof of this completeness which has the advantage of being significantly more straightforward and elegant than it predecessors. 
Table 1. The complete family of $\hbar$-independent additive shape-invariant superpotentials.

\begin{tabular}{ll}
\hline Name & Superpotential \\
\hline Harmonic Oscillator & $\frac{1}{2} \omega x$ \\
Coulomb & $\frac{e^{2}}{2(\ell+1)}-\frac{\ell+1}{r}$ \\
3-D oscillator & $\frac{1}{2} \omega r-\frac{\ell+1}{r}$ \\
Morse & $A-B e^{-x}$ \\
Rosen-Morse I & $-A \cot x-\frac{B}{A}$ \\
Rosen-Morse II & $A \tanh x+\frac{B}{A}$ \\
Eckart & $-A \operatorname{coth} x+\frac{B}{A}$ \\
Scarf I & $A \tan x-B \sec x$ \\
Scarf II & $A \tanh x+B \operatorname{sech} x$ \\
Gen. Pöschl-Teller & $A \operatorname{coth} x-B \operatorname{cosech} x$ \\
\hline
\end{tabular}

\subsection{New Proof of Completeness of the Conventional Shape-Invariant Superpotentials}

Because of additive shape invariance, the dependence of $W$ on $a$ and $\hbar$ is through the linear combination $a+\hbar$; therefore, the derivatives of $W$ with respect to $a$ and $\hbar$ are related by: $\frac{\partial W(x, a+\hbar)}{\partial \hbar}=\frac{\partial W(x, a+\hbar)}{\partial a}$. Since Equation (7) must hold for an arbitrary value of $\hbar$, if we assume that $W$ does not depend explicitly on $\hbar$, we can expand in powers of $\hbar$, and the coefficient of each power must separately vanish. Expanding the right hand side in powers of $\hbar$ yields

$$
\begin{aligned}
\mathcal{O}(\hbar) & \Rightarrow W \frac{\partial W}{\partial a}-\frac{\partial W}{\partial x}+\frac{1}{2} \frac{d g(a)}{d a}=0 \\
\mathcal{O}\left(\hbar^{2}\right) & \Rightarrow \frac{\partial}{\partial a}\left(W \frac{\partial W}{\partial a}-\frac{\partial W}{\partial x}+\frac{1}{2} \frac{d g(a)}{d a}\right)=0 \\
\mathcal{O}\left(\hbar^{n}\right) & \Rightarrow \frac{\partial^{n}}{\partial a^{n-1} \partial x} W(x, a)=0, \quad n \geq 3
\end{aligned}
$$

Thus, all conventional additive shape invariant superpotentials are solutions of the above set of non-linear partial differential equations [46,47]. Although this represents an infinite set, note that if equations of $\mathcal{O}(\hbar)$ and $\mathcal{O}\left(\hbar^{3}\right)$ are satisfied, all others automatically follow. Therefore, we proceed to find all possible solutions to the two partial differential equations:

$$
W \frac{\partial W}{\partial a}-\frac{\partial W}{\partial x}+\frac{1}{2} \frac{d g(a)}{d a}=0
$$

and

$$
\frac{\partial^{3}}{\partial a^{2} \partial x} W(x, a)=0
$$

In doing so, we derive a new proof that the superpotentials shown in Table 1 are the only possible solutions.

The general solution to Equation (51) is

$$
W(x, a)=a \cdot X_{1}(x)+X_{2}(x)+u(a)
$$


Therefore, to generate all shape invariant superpotentials, we need to determine all possible combinations of $u(a), X_{1}(x)$, and $X_{2}(x)$ that satisfy Equation (50). We will ignore the case when both $X_{1}(x)$, and $X_{2}(x)$ are constants, as this corresponds to a flat potential with no $x$-dependence. We will also ignore the case in which $X_{1}(x)$, and $X_{2}(x)$ are linearly dependent on each other; i.e., $X_{2}(x)=\alpha X_{1}(x)+\beta$. In this case, $W(x)=a X_{1}(x)+\alpha X_{1}(x)+\beta+u(a)=(a+\alpha) X_{1}(x)+\beta+u(a)$. If we redefine $\tilde{a} \equiv a+\alpha$, this case becomes equivalent to a superpotential with a shifted parameter and constant $X_{2}$ which will be considered shortly. We can therefore assume that $X_{1}$ and $X_{2}$ are linearly independent of each other without loss of generality. Note that from here onward, we will use lower case Greek letters to denote constants that are independent of both $a$ and $x$.

To determine $W(x, a)$, we first focus on determining $u(a)$. To do so, we take two derivatives of (50) with respect to $a$. This leads to the following differential equation:

$$
3 \dot{W} \ddot{W}+W \dddot{W}+\ddot{W}^{\prime}+\frac{1}{2} \dddot{g}=0
$$

where dots and primes represent derivatives taken with respect to $a$ and $x$ respectively. Since $\frac{\partial^{3}}{\partial a^{2} \partial x} W(x, a)=0$, this simplifies to:

$$
3 \dot{W} \ddot{W}+W \dddot{W}+\frac{1}{2} \dddot{g}=0
$$

Inserting the form of the general solution (52) into (53) yields

$$
X_{1}(3 \ddot{u}+a \dddot{u})+X_{2} \dddot{u}=H(a)
$$

where $H(a)=-\left(3 \dot{u} \ddot{u}+u \dddot{u}+\frac{1}{2} \dddot{g}\right)$ is a function of $a$, and is independent of $x$. Since $X_{1}$ and $X_{2}$ are linearly independent, we find that there are only three possible ways for the left-hand-side of Equation (54) to be independent of $x$ :

- Case 1: $X_{1}$ is a constant and $\dddot{u}=0$;

- Case 2: $X_{2}$ is a constant and $3 \ddot{u}+a \dddot{u}=0$;

- Case 3: Neither $X_{1}$ nor $X_{2}$ are not constants, but $3 \ddot{u}+a \dddot{u}=0$ and $\dddot{u}=0$.

For each of these cases we can determine the form of $u(a)$. Then we can determine $X_{1}(x)$ and $X_{2}(x)$ for these three cases. This we do by taking two derivatives of (50), this time one with respect to $a$ and another with respect to $x$. This yields:

$$
2 \dot{W} \dot{W}^{\prime}+W^{\prime} \ddot{W}-\dot{W}^{\prime \prime}=0
$$

Inserting the form of the general solution (52) into (55) yields

$$
2\left(X_{1}+\dot{u}\right) X_{1}^{\prime}+\left(a X_{1}^{\prime}+X_{2}^{\prime}\right) \ddot{u}-X_{1}^{\prime \prime}=0
$$

Now, we will analyze each of the three cases in detail. 


\subsubsection{Case 1: $X_{1}$ Is a Constant and $\dddot{u}=0$}

Let $X_{1}=\alpha$. Since $X_{2}$ cannot be a constant as well, Equation (54) requires $\dddot{u}=0$. This leads to $u(a)=\gamma a^{2}+\mu a+\nu$ for some arbitrary constants $\alpha, \gamma, \mu$, and $\nu$. Inserting $X_{1}$ and $u$ into Equation (52) yields $W(x, a)=X_{2}(x)+\gamma a^{2}+\eta a+\nu$, where $\eta=\alpha+\mu$.

We now find $X_{2}$ by inserting the above $W$ into Equation (50). This yields

$$
\left(X_{2}+\gamma a^{2}+\eta a+\nu\right)(2 a \gamma+\eta)-\frac{d X_{2}}{d x}=-\frac{1}{2} \frac{d g}{d a}
$$

or equivalently,

$$
2 a^{3} \gamma^{2}+3 a^{2} \eta \gamma+a\left(2 X_{2} \gamma+2 \nu \gamma+\eta^{2}\right)+X_{2} \eta+\eta \nu-X_{2}^{\prime}=h(a)
$$

where $h(a)=-\frac{1}{2} \frac{d g}{d a}$.

Since $X_{2}(x)$ is independent of $a$, and the left side of (57) is a sum of four linearly independent functions of $a\left(a^{0}, a^{1}, a^{2}\right.$, and $\left.a^{3}\right)$, and the term $h(a)$ on the right-hand-side is independent of $x$, the coefficient of each power of $a$ must separately be independent of $x$. The linear term in $a$ therefore requires that $2 X_{2} \gamma+2 \nu \gamma+\eta^{2}$ be independent of $x$. Since a constant $X_{2}$ leads to a trivial solution, we must have $\gamma=0$. The remaining $x$-dependent terms on the left side of (57), $X_{2} \eta-X_{2}^{\prime}$ must be a constant:

$$
\eta X_{2}-X_{2}^{\prime}=\beta
$$

The solution depends on the value of the constants $\eta$ and $\beta$.

- Case 1A: $\eta=0, \beta=0$. In this case, $X_{2}^{\prime}=0$, which is a trivial solution;

- Case 1B: $\eta=0, \beta \neq 0$. In this case, $X_{2}=-\beta x+\xi$, so $W=-\beta x+\xi+\nu$. Defining $\beta=-\frac{1}{2} \omega$ yields the harmonic oscillator superpotential;

- Case 1C: $\eta \neq 0$. The solution is then $X_{2}(x)=-\frac{\beta}{\eta}+\frac{1}{\eta} e^{\eta\left(x-x_{0}\right)}, u=\mu a+\nu, X_{1}=\alpha$. Therefore, $W=\left(\nu-\frac{\beta}{\eta}+\eta a\right)+\frac{1}{\eta} e^{\eta\left(x-x_{0}\right)}$. For $\eta=-1$, this yields $W=A-B e^{-x}$, where $A \equiv \nu+\beta-a$ and $B \equiv e^{x_{0}}$. This is the Morse superpotential. Note that $A$ decreases as $a$ increases, and hence signals a finite number of eigenstates [48].

\subsubsection{Case 2: $X_{2}$ Is Constant}

In this case, let $X_{2}=\alpha$; then Equation (54) requires $3 \ddot{u}+a \dddot{u}=0$. This yields $u(a)=\mu a+\nu+\frac{\gamma}{a}$. We now insert this form of $u$ and $X_{2}=\alpha$ into (56) to get an ordinary differential equation in $x$ for $X_{1}$ :

$$
2\left(X_{1}+\mu-\frac{\gamma}{a^{2}}\right) X_{1}^{\prime}+2 X_{1}^{\prime} \frac{\gamma}{a^{2}}-X_{1}^{\prime \prime}=0
$$

or equivalently,

$$
2 X_{1} X_{1}^{\prime}+2 \mu X_{1}^{\prime}-X_{1}^{\prime \prime}=0
$$

Integrating it once, we get

$$
X_{1}^{2}+2 \mu X_{1}-X_{1}^{\prime}=\beta
$$


This equation can be simplified by setting $\tilde{X}_{1}=X_{1}+\mu$. This leads to

$$
\tilde{X}_{1}^{2}-\tilde{X}_{1}^{\prime}=\left(\beta+\mu^{2}\right) \equiv \theta
$$

The solutions for $\tilde{X}_{1}$ depend on the constant $\theta$.

- Case 2A: $\theta=0$, In this case, $\tilde{X}_{1}=-\frac{1}{x-x_{0}}$. The whole superpotential is then given by

$$
\begin{aligned}
W(x, a) & =a\left(-\frac{1}{x-x_{0}}-\mu\right)+\alpha+\left(\mu a+\nu+\frac{\gamma}{a}\right) \\
& =-\frac{a}{x-x_{0}}+(\alpha+\nu)+\frac{\gamma}{a}
\end{aligned}
$$

Setting $\alpha+\nu=0$ and $x_{0}=0$, and identifying $x \equiv r, a \equiv \ell+1$ and $\gamma=e^{2} / 2$, we get $W(r, \ell)=\frac{e^{2}}{2(\ell+1)}-\frac{\ell+1}{r}$ : the superpotential for Coulomb [49];

- Case 2B: $\theta>0$. In this case, we have either $\tilde{X}_{1}=-\sqrt{\theta} \operatorname{coth}(\sqrt{\theta} x-\eta)$ (Eckart) or $\tilde{X}_{1}=-\sqrt{\theta} \tanh (\sqrt{\theta} x-\eta)$ (Rosen-Morse II). In the first case, the superpotential is given by $W(x, a)=a(-\sqrt{\theta} \operatorname{coth}(\sqrt{\theta} x)-\mu)+\alpha+\left(\mu a+\nu+\frac{\gamma}{a}\right) \equiv-A \operatorname{coth}(\sqrt{\theta} x)+\frac{B}{a}$, where we have set $\gamma=B,(\alpha+\nu)=0, \theta=1$ and $\eta=0$. This is the well known Eckart potential. Similarly, the other solution with $\tanh x$ generates Rosen-Morse II;

- Case 2C: $\theta<0$. In this case, we obtain $\tilde{X}_{1}=-\sqrt{|\theta|} \cot (\sqrt{|\theta|} x-\eta)$. An analysis similar to the previous case generates the superpotential for Rosen-Morse I.

\subsubsection{Case 3: $X_{1}$ and $X_{2}$ Are not Constant, but $3 \ddot{u}+a \dddot{u}=0$ and $\dddot{u}=0$}

In this case, since $\dddot{u}=0$, and $3 \ddot{u}+a \dddot{u}=0$, we have $\ddot{u}=0$. Therefore $u(a)=\mu a+\nu$. In this case, Equation (56) yields

$$
2\left(X_{1}+\mu\right) X_{1}^{\prime}-X_{1}^{\prime \prime}=0
$$

Integrating,

$$
X_{1}^{2}+2 \mu X_{1}-X_{1}^{\prime}=\beta
$$

Thus, again we have

$$
\tilde{X}_{1}^{2}-\tilde{X}_{1}^{\prime}=\left(\beta+\mu^{2}\right) \equiv \theta
$$

Note that this is the same differential equation as (60) and will therefore give the same solutions for $X_{1}$ as Case 2. However, in this case, $u=\mu a+\nu$ (this is equivalent to choosing $\gamma=0$ in Case 2) and $X_{2}$ is not constant. Instead, in each case we must plug our solutions for $u(a)$ and $X_{1}(x)$ into Equation (50), which yields

$$
\left(a X_{1}+X_{2}+\mu a+\nu\right)\left(X_{1}+\mu\right)-a X_{1}^{\prime}-X_{2}^{\prime}=h(a)
$$

This equation is again simplified by setting $\tilde{X}_{1}=X_{1}+\mu$, which yields

$$
\left(a \tilde{X}_{1}+X_{2}+\nu\right) \tilde{X}_{1}-a \tilde{X}_{1}^{\prime}-X_{2}^{\prime}=h(a)
$$


Since $\tilde{X}_{1}$ and $X_{2}$ are independent of $a$, the terms linear in $a$ and the terms independent of $a$ on the left side of this equation must each separately be independent of $x$. Therefore,

$$
\begin{gathered}
\left(\tilde{X}_{1}\right)^{2}-\tilde{X}_{1}^{\prime}=\theta, \text { and } \\
X_{2} \tilde{X}_{1}+\nu \tilde{X}_{1}-X_{2}^{\prime}=\xi
\end{gathered}
$$

For different values of $\theta$, we get different superpotentials:

- Case 3A: $\theta=0$. We again get $\tilde{X}_{1}=-\frac{1}{x}$, where with an appropriate choice for the origin we have set $x_{0}=0$. Equation (65) for $X_{2}$ becomes

$$
\left(X_{2}+\nu\right)+x X_{2}^{\prime}=-\xi x
$$

Its solution is $X_{2}=-\frac{1}{2} \xi x+\frac{\sigma}{x}-\nu$. With the identification $x \rightarrow r, \xi \rightarrow-\omega, a-\sigma \rightarrow \ell+1$, we get $W(r, \ell)=\frac{1}{2} \omega r-\frac{\ell+1}{r}$, the superpotential for the 3D-harmonic oscillator;

- Case 3B: $\theta>0$. As seen before, $\theta>0$ implies that $\tilde{X}_{1}=-\sqrt{\theta} \operatorname{coth}(\sqrt{\theta} x-\eta)$ or $\tilde{X}_{1}=$ $-\sqrt{\theta} \tanh (\sqrt{\theta} x-\eta)$. By translation and scaling of $x$, we can simplify the first solution to $\tilde{X}_{1}=-\operatorname{coth} x$. Substituting $\tilde{X}_{1}$ in Equation (65), we get

$$
-\operatorname{coth} x \tilde{X}_{2}-\tilde{X}_{2}^{\prime}=\xi
$$

where we have set $\tilde{X}_{2}=X_{2}+\nu$. The solution to the homogeneous equation is $\tilde{X}_{2}=\frac{\sigma}{\sinh x}$, and the particular solution is $\xi \operatorname{coth} x$. Hence $X_{2}=\frac{\sigma}{\sinh x}+\xi \operatorname{coth} x-\nu$. Thus, the superpotential is given by $W(a, x)=(\xi-a) \operatorname{coth} x+\frac{\sigma}{\sinh x}$, the General Pöschl-Teller potential given in Table 1 . The second solution generates the Scarf II potential;

- Case 3C: $\theta<0$. A similar analysis for this case leads to Scarf I as the corresponding shape invariant superpotential.

Thus, we have generated all the superpotentials of Table 1 and shown that these are the only possible $\hbar$-independent solutions to the additive shape invariant condition.

\section{3. $\hbar$-Dependent Superpotentials}

In the previous section we generated the complete list of additive shape-invariant superpotentials that do not depend explicitly on $\hbar$. However, a new class of superpotentials was discovered by Quesne [50,51]. It has been shown [46] that these "extended" superpotentials obey the shape invariance condition in the form of Equation (7) only when $W$ is allowed to depend explicitly on $\hbar$. While this dependence is frequently ignored by the conventional notation that sets $\hbar=1$, we will show that this constraint results in important consequences for the energy spectrum of the resulting potentials. In each case, the new potential is isospectral with a potential that arises from one of the "conventional" superpotentials listed in Table 1. Authors of [52-54] have added an infinite number of potentials that belong in this class, and extended shape invariant potentials continue to be objects of intense research $[55,56]$. 
We now extend our formalism to include "extended" superpotentials that contain $\hbar$ explicitly. To do so, we expand the superpotentials in powers of $\hbar$. Hence, we define

$$
W(x, a, \hbar)=\sum_{n=0}^{\infty} \hbar^{n} W_{n}(x, a)
$$

We will now substitute Equation (68) into the shape invariance condition given in Equation (7), for which we compute $\left.\frac{\partial W}{\partial x}\right|_{a=a_{0}}$ and $W^{2}\left(x, a_{0}, \hbar\right)$. We obtain

$$
\left.\frac{\partial W}{\partial x}\right|_{a=a_{0}}=\sum_{n=0}^{\infty} \hbar^{n} \frac{\partial W_{n}\left(x, a_{0}\right)}{\partial x}
$$

and

$$
W^{2}\left(x, a_{0}, \hbar\right)=\sum_{l=0}^{\infty} \sum_{k=0}^{\infty} \hbar^{k+l} W_{k} W_{l}
$$

Since $a_{1}=a_{0}+\hbar, W\left(x, a_{1}, \hbar\right)=W\left(x, a_{0}+\hbar, \hbar\right)$. Expanding in powers of $\hbar$,

$$
W\left(x, a_{1}, \hbar\right)=\sum_{m=0}^{\infty} \sum_{k=0}^{m} \frac{\hbar^{m}}{k !} \frac{\partial^{k} W_{m-k}}{\partial a^{k}} ; \text { and } W^{2}\left(x, a_{1}, \hbar\right)=\sum_{n=0}^{\infty} \sum_{s=0}^{n} \sum_{k=0}^{s} \frac{\hbar^{n}}{(n-s) !} \frac{\partial^{n-2}\left(W_{k} W_{s-k}\right)}{\partial a^{n-2}}
$$

Similarly,

$$
\left.\frac{\partial W}{\partial x}\right|_{a=a_{1}}=\sum_{m=0}^{\infty} \sum_{k=0}^{m} \frac{\hbar^{m}}{k !} \frac{\partial^{k+1} W_{m-k}}{\partial a^{k} \partial x}
$$

We substitute these into Equation (7) and stipulate that the equation hold for any value of $\hbar$. After some significant algebraic manupulation we find that the following equation must be true separately for each positive integer value of $n$ :

$$
\begin{array}{r}
\sum_{k=0}^{n} W_{k} W_{n-k}+\frac{\partial W_{n-1}}{\partial x}-\sum_{s=0}^{n} \sum_{k=0}^{s} \frac{1}{(n-s) !} \frac{\partial^{n-s}}{\partial a^{n-s}} W_{k} W_{s-k} \\
+\sum_{k=1}^{n} \frac{1}{(k-1) !} \frac{\partial^{k}}{\partial a^{k-1} \partial x} W_{n-k}-\left(\frac{1}{n !} \frac{\partial^{n} g}{\partial a^{n}}\right)=0
\end{array}
$$

For $n=1$, we obtain

$$
2 \frac{\partial W_{0}}{\partial x}-\frac{\partial}{\partial a}\left(W_{0}^{2}+g\right)=0
$$

This equation is identical to Equation (50) for $\hbar$-independent $W$ 's. We have already found a set of solutions for Equation (70) that includes all known $\hbar$-independent superpotentials. The extended cases [50,51] are solutions to (69) as well, as shown in [46]. Note that Equation (69) provides a consistency condition for all $\hbar$-dependent potentials; however, these are not easy to solve to determine new potentials. 
Additionally, Equation (70) provides a constraint for the possible energy spectra of the extended potentials. from Equation (70), the function $g(a)$ is given by

$$
\begin{aligned}
\frac{\partial g}{\partial a} & =2 \frac{\partial W_{0}}{\partial x}-\frac{\partial W_{0}^{2}}{\partial a} \\
g(a) & =\int d a\left[2\left(\frac{\partial W_{0}}{\partial x}\right)-W_{0}^{2}\right]
\end{aligned}
$$

Thus, the function $g(a)$, and hence the energy of the system, is given entirely in terms of the $\hbar$-independent part of the superpotential. Hence, the eigenvalues are not affected by the $\hbar$-dependent extension of the superpotential.

Thus far, each of the known extended potentials contains a solution from Table 1 as the $\hbar$-independent term of the superpotential $W_{0}$. Therefore, each of the expanded potentials is isospectral with its corresponding conventional potential. Future possibilities for finding new shape-invariant superpotentials fall into one of two categories:

1. Further extended superpotentials may be found based on the conventional superpotentials. In this case, the potentials derived from the extended superpotential will be isospectral with the potentials derived from the corresponding conventional superpotential;

2. While $W_{0}$ is required to satisfy Equation (70), which is equivalent to Equation (50) for $\hbar$-independent $W$ 's, $W_{0}$ is not required to satisfy Equation (48). Rather, the additional constraints for an extended $W$ are supplied by Equation (69). It therefore may be possible to find an $\hbar$-dependent superpotential whose $\hbar$-independent term $W_{0}$ is not equivalent to a conventional superpotential. Intriguingly, it therefore may still be possible to discover shape-invariant systems with new energy spectra.

\section{Summary and Conclusions}

While supersymmetric quantum mechanics began as a simplified model to account for dynamical symmetry breaking, the application of this formalism to quantum mechanics has become an important field in its own right. In this manuscript we have reviewed research on supersymmetric quantum mechanics with a particular emphasis on the property of shape invariance. As we have shown, shape invariance is a sufficient condition for exact solvability of quantum mechanical problems; i.e., given a superpotential with shape invariance, all its eigenvalues and eigenfunctions can be determined analytically.

However, in its traditional form, the shape invariance condition Equation (7) is a difference-differential equation and is difficult to solve. It has recently been established that for additive shape-invariant superpotentials that do not explicitly depend on $\hbar$, this condition can be written as a set of local partial differential equations [45-47]. The solution to these equations showed that the list of such superpotentials was indeed complete. In this manuscript, we have presented a more straightforward proof of this result.

Since 2008, new sets of additive shape invariant potentials have been discovered [50-54]. We have reviewed the development of these "extended" shape-invariant systems and have provided an infinite 
set of partial differential equations that all extended potentials (where superpotentials are inherently functions of $\hbar$ ) must obey [46,47]. We have also discussed the constraints placed on the energy spectra of these extended potentials as well as possibilities for finding additional as-yet-undiscovered cases of additive shape invariance.

It may also be possible to extend this method to other forms of shape invariance such as multiplicative or cyclic. For these, the potentials are generally not available in terms of known functions, except in very special cases ( $N=2$ for cyclic and limiting cases for multiplicative). It remains to be shown whether the shape invariance condition for these classes can be transformed from a difference-differential equation into a set of partial differential equations and be subjected to similar analysis.

\section{Acknowledgement}

This research was supported by an award from Research Corporation for Science Advancement.

\section{References and Notes}

1. Darboux, G. Leçons sur la Théorie Général des Surfaces, 2nd ed.; Gauthier-Villars: Paris, France, 1912.

2. Schrödinger, E. A method of determining quantum-mechanical eigenvalues and eigenfunctions. Proc. R. Ir. Acad. 1940, A46, 9-16.

3. Schrödinger, E. Further studies on solving eigenvalue problems by factorization. Proc. $R$. Ir. Acad. 1941, A46, 183-206.

4. Schrödinger, E. The factorization of the hypergeometric equation. Proc. R. Ir. Acad. 1941, A47, 53-54.

5. Infeld, L.; Hull, T.E. The factorization method. Rev. Mod. Phys. 1951, 23, 21-68.

6. Witten, E. Dynamical breaking of supersymmetry. Nucl. Phys. 1981, B185, 513-554.

7. Solomonson, P.; Van Holten, J.W. Fermionic coordinates and supersymmetry in quantum mechanics. Nucl. Phys. 1982, B196, 509-531.

8. Cooper, F.; Freedman, B. Aspects of supersymmetric quantum mechanics. Ann. Phys. 1983, 146, 262-288.

9. Note the constant $-\frac{1}{2} \hbar \omega$ has been added to the usual harmonic oscillator potential to insure that the groundstate energy of the system remains at zero. This constant allows us to factorize the Hamiltonian $H_{-}$as a product of operators $\mathcal{A}^{+}$and $\mathcal{A}^{-}$.

10. Cooper, F.; Khare, A.; Sukhatme, U. Supersymmetry in Quantum Mechanics; World Scientific: Singapore, 2001.

11. Gangopadhyaya, A.; Mallow, J.; Rasinariu, C. Supersymmetric Quantum Mechanics: An Introduction; World Scientific: Singapore, 2010.

12.

$H_{+}\left(\mathcal{A}^{-} \psi_{n+1}^{(-)}\right)=\mathcal{A}^{-} \mathcal{A}^{+}\left(\mathcal{A}^{-} \psi_{n+1}^{(-)}\right)=\mathcal{A}^{-}\left(\mathcal{A}^{+} \mathcal{A}^{-} \psi_{n+1}^{(-)}\right)=\mathcal{A}^{-} H_{-}\left(\psi_{n+1}^{(-)}\right)=E_{n+1}^{-}\left(\mathcal{A}^{-} \psi_{n+1}^{(-)}\right)$.

Thus, $\left(\mathcal{A}^{-} \psi_{n+1}^{(-)}\right)$is an eigenstate of $H_{+}$with an eigenvalue $E_{n+1}^{-}$.

13. $\left.\left\langle\psi\left|H_{\mp}\right| \psi\right\rangle=\left\langle\psi\left|A^{ \pm} A^{\mp}\right| \psi\right\rangle=\left|A^{\mp}\right| \psi\right\rangle\left.\right|^{2} \geq 0$. 
14. Bender, C.M.; Boettcher, S. Real spectra in non-hermitian hamiltonians having PT symmetry. Phys. Rev. Lett. 1998, 80, 5243-5246.

15. Bender, C.M.; Brody, D.C.; Jones, H.F. Complex extension of quantum mechanics. Phys. Rev. Lett. 2002, 89, 270401:1-270401:4.

16. Bender, C.M.; Berry, M.V.; Mandilara, A. Generalized PT symmetry and real spectra. J. Phys. A 2002, 35, L467-L471.

17. Znojil, M. SI potentials with PT symmetry. J. Phys. A 2000, 33, L61-L62.

18. Levai, Z. Exact analytic study of the PT-symmetry-breaking mechanism. Czech. J. Phys. 2004, $54,77-84$.

19. Znojil, M. Matching method and exact solvability of discrete Pt-symmetric square wells. J. Phys. A 2006, 39, 10247-10261.

20. Quesne, C.; Bagchi, B.; Mallik, S.; Bila, H.; Jakubsky, V. PT supersymmetric partner of a short-range square well. Czech. J. Phys. 2005, 55, 1161-1166.

21. Bagchi, B.; Quesne, C.; Roychoudhury, R. Isospectrality of conventional and new extended potentials, second-order supersymmetry and role of PT Symmetry. Pramana 2009, 73, 337-347.

22. Miller, W., Jr. Lie Theory and Special Functions (Mathematics in Science and Engineering); Academic Press: New York, NY, USA, 1968.

23. Gendenshtein, L.E. Derivation of exact spectra of the schrodinger equation by means of supersymmetry. JETP Lett. 1983, 38, 356-359.

24. Gendenshtein, L.E.; Krive, I.V. Supersymmetry in quantum mechanics. Sov. Phys. Usp. 1985, 28, 645-666.

25. Barclay, D.; Dutt, R.; Gangopadhyaya, A.; Khare, A.; Pagnamenta, A.; Sukhatme, U. New exactly solvable hamiltonians: Shape invariance and self-similarity. Phys. Rev. A 1993, 48, 2786-2797.

26. Spiridonov, V.P. Exactly solvable potentials and quantum algebras. Phys. Rev. Lett. 1992, 69, 398-401.

27. Sukhatme, U.P.; Rasinariu, C.; Khare, A. Cyclic shape invariant potentials. Phys. Lett. 1997, A234, 401-409.

28. Gangopadhyaya, A.; Mallow, J.V.; Sukhatme, U.P. Supersymmetry and Integrable Models: Proceedings of Workshop on Supersymmetry and Integrable Models; Aratyn, H., Imbo, T.D., Keung, W.-Y., Sukhatme, U., Eds.; Springer-Verlag: Berlin, Germany, 1997.

29. Balantekin, A.B. Algebraic approach to shape invariance. Phys. Rev. A 1998, 57, 4188-4191.

30. Gangopadhyaya, A.; Mallow, J.V.; Sukhatme, U.P. Translational shape invariance and the inherent potential algebra. Phys. Rev. A 1998, 58, 4287-4292.

31. Chaturvedi, S.; Dutt, R.; Gangopadhyaya, A.; Panigrahi, P.; Rasinariu, C.; Sukhatme, U. Algebraic shape invariant models. Phys. Lett. 1998, A248, 109-113.

32. Balantekin, A.; Candido Ribeiro, M.; Aleixo, A. Algebraic nature of shape-invariant and self-similar potentials. J. Phys. A 1999, 32, 2785-2790.

33. We assume that as $a_{0} \rightarrow a_{1}$, the supersymmetry remains unbroken.

34. Dutt, R.; Khare, A.; Sukhatme, U. Supersymmetry, shape invariance and exactly solvable potentials. Am. J. Phys. 1998, 56, 163. 
35. Cooper, F.; Ginocchio, J.; Khare, A. Relationship between supersymmetry and solvable potentials. Phys. Rev. D 1987, 36, 2458.

36. In the last line we have used the fact that $\left(i \partial_{\phi}\right) \mathrm{e}^{-i s \phi}=\mathrm{e}^{-i s \phi}\left(i \partial_{\phi}+s\right)$. This implies that for any analytical function $\chi(x)$, we have $\chi\left(i \partial_{\phi}\right) \mathrm{e}^{-i s \phi}=\mathrm{e}^{-i s \phi} \chi\left(i \partial_{\phi}+s\right)$.

37. Veselov, A.P.; Shabat, A.B. Dressing chains and spectral theory of the Schrödinger operator. Funct. Anal. Appl. 1993, 27, 81-96.

38. These constraints are: $a d-b c=1 ; a+d=2 \cos (2 \pi j / k), j=0,1, \ldots, k-1$.

39. We have used $\left[-\frac{i}{s} \partial_{\phi}, \mathrm{e}^{ \pm i s \phi}\right]= \pm \mathrm{e}^{ \pm i s \phi}$.

40. Dutt, R.; Gangopadhyaya, A.; Rasinariu, C.; Sukhatme, U. Coordinate realizations of deformed Lie algebras with three generators. Phys. Rev. A 1999, 60, 3482-3486.

41. Rocek, M. Representation theory of the nonlinear SU (2) algebra. Phys. Lett. B 1991, 255, 554-557.

42. Adams, B.G.; Cizeka, J.; Paldus, J. Lie algebraic methods and their applications to simple quantum systems. Advances in Quantum Chemistry, 19th ed.; Academic Press: New York, NY, USA, 1987.

43. In some cases these are additive constants and subtracted away. If we provide a common floor to all potentials, demanding that their groundstate energies be zero, we will find that all known solvable potentials pick up a $\hbar$ dependent term.

44. Shabat, A. The infinite-dimensional dressing dynamical system. Inverse Probl. 1992, 8, 303-308.

45. Gangopadhyaya, A.; Mallow, J.V. Generating shape invariant potentials. Int. J. Mod. Phys. A 2008, 23, 4959-4978.

46. Bougie, J.; Gangopadhyaya, A.; Mallow, J.V. Generation of a complete set of additive shape-invariant potentials from an euler equation. Phys. Rev. Lett. 2010, 210402:1-210402:4.

47. Bougie, J.; Gangopadhyaya, A.; Mallow, J.V. Method for generating additive shape invariant potentials from an euler equation. J. Phys. A 2012, 44, 275307:1-275307:19.

48. Normalizability of the groundstate $\left(\sim e^{-\int^{x} W(A, B, y) d y}\right)$ requires that $A$ be greater than zero. Since an increase in $a$ decreases $A$, there can only be a finite number of increases.

49. By substituting $W(x, a)=-\frac{a}{x-x_{0}}+(\alpha+\nu)+\frac{\gamma}{a}$ into Equation (50) we find that shape invariance requires that $\alpha+\nu=0$.

50. Quesne, C. Exceptional orthogonal polynomials, exactly solvable potentials and supersymmetry. J. Phys. A 2008, 41, 392001:1-392001:6.

51. Quesne, C. Solvable rational potentials and exceptional orthogonal polynomials in supersymmetric quantum mechanics. Sigma 2009, 5, 084:1-084:24.

52. Odake, S.; Sasaki, R. Infinitely many shape invariant discrete quantum mechanical systems and new exceptional orthogonal polynomials related to the wilson and Askey-Wilson polynomials. Phys. Lett. B 2009, 682, 130-136.

53. Odake, S.; Sasaki, R. Another set of infinitely many exceptional $\left(X_{\ell}\right)$ laguerre polynomials. Phys. Lett. B 2010, 684, 173-176.

54. Tanaka, T. N-fold supersymmetry and quasi-solvability associated with X-2-laguerre polynomials. J. Math. Phys. 2010, 51, 032101:1-032101:20. 
55. Sree Ranjani, S.; Panigrahi, P.; Khare, A.; Kapoor, A.; Gangopadhyaya, A. Exceptional orthogonal polynomials, QHJ formalism and SWKB quantization condition. J. Phys. A 2012, 055210:1-055210:10.

56. Shiv Chaitanya, K.; Sree Ranjani, S.; Panigrahi, P.; Radhakrishnan, R.; Srinivasan, V. Exceptional polynomials and SUSY quantum mechanics. Available online: http://arxiv.org/pdf/1110.3738.pdf (accessed on 2 August 2012).

(C) 2012 by the authors; licensee MDPI, Basel, Switzerland. This article is an open access article distributed under the terms and conditions of the Creative Commons Attribution license (http://creativecommons.org/licenses/by/3.0/). 